\title{
The rise and fall of the compact jet in GRO J1655-40
}

\section{Simone Migliari*}

Center for Astrophysics and Space Sciences, University of California San Diego, 9500 Gilman

Dr., La Jolla, CA 92093, USA.

E-mail: migliari@ucsd.edu

\section{John Tomsick}

Space Sciences Laboratory, University of California Berkeley, 7 Gauss Way, Berkeley, CA

94720-7450, USA.

E-mail: jtomsickessl.berkeley.edu

\section{Sera Markoff}

Astronomical Institute 'Anton Pannekoek', University of Amsterdam, 403 Kruislaan, 1098 SJ,

Amsterdam, the Netherlands.

E-mail: seraescience.uva.nl

\section{Emrah Kalemci}

Sabanci University, Orhanli-Tuzla, Istanbul, 34956, Turkey

E-mail: ekalemciesabanciuniv.edu

In this work, we present some preliminary results on a multi-wavelength (radio/infrared/optical/X-ray) study of GRO J1655-40 during its 2005 outburst. We focus on the broadband spectral energy distribution during the different stages of the outburst. In particular, using this unprecedented coverage, and especially thanks to the new constraints given in the mid-IR by Spitzer, we can test the physical self-consistent disk-jet model during the hard state, where the source shows radio emission from a compact jet. The hard state broadband spectra of the observations during the decay of the outburst, are fairly well fit using the jet model with parameters overall similar to those found for Cyg X-1 and GX 339-4 in a previous work. However, we find that, compared to the other two BHs, GRO J1655-40 has a much higher jet power (at least a factor of 3), and that, most notably, the model seems to underestimate the radio emission.

VI Microquasar Workshop: Microquasars and Beyond

September 18-22 2006

Società del Casino, Como, Italy

\footnotetext{
* Speaker.
} 


\section{Introduction}

Galactic black hole (BH) X-ray binaries (XRB) spend most of their time in quiescence, but occasionally show transient outbursts resulting in an increase in luminosity of many orders of magnitude at all wavelengths. These outbursts are explained as the result of disk instabilities, possibly due to a dramatic increase of mass accretion rate. By means of multiwavelength campaigns, especially in more recent years, we are obtaining unprecedented information on the physical processes in the binary systems. Each observing band can give us insights into a specific radiative component of the X-ray binary: in the radio band we observe synchrotron radiation from the jet outflow, in $\mathrm{X}$ rays we observe the accretion disk and the Comptonizing corona and/or the base of the jet, and in the optical/IR band the superposition of the companion star, the outer regions of the accretion disk, and the jet.

The different stages of an outburst can be described in terms of transitions between 'X-ray states'. The definition of the X-ray states is based on X-ray spectral and temporal behaviour and it is, in its details, still an area of controversy. In this work, we will follow the nomenclature in [17][16]. These X-ray spectral states are also associated with a specific radio (jet) behaviour (see [3] for a review). During the hard state, an optically thick synchrotron radio emission (a compact jet) is observed. During the thermal state the radio emission is quenched, likely associated to the suppression of the compact jet. The Steep Power Law state, and in general the transition hard-tothermal state, seems to be associated with radio optically thin flares, signature of the ejection of transient jets.

The BH XRB GRO J1655-40 was the second superluminal jet source discovered in our galaxy [22] [11]. The mass of the compact object has been dynamically estimated to be $M=6.3 \pm 0.5 M_{\odot}$, and from the optical photometry also an inclination of the binary of $70^{\circ} .2 \pm 1^{\circ} .9$ has been derived [6]. From Very Long Baseline Interferometry (VLBI) observations of the transient radio jets of GRO J1655-40, [11] derived, using a distance of $3.2 \mathrm{kpc}$ (in agreement with previous estimates: [22]), a jet axis inclination of $\sim 85^{\circ}$ to the line of sight, with a possible precession of the jet around the axis of $\sim 2^{\circ}$. Foellmi et al. [5], based on the estimated optical absorption towards GRO J165540 , have recently put an upper limit to the distance of the source of $\sim 1.7 \mathrm{kpc}$. With this new distance, the source would not be anymore superluminal. Note that, using a lower distance of 1.7 $\mathrm{kpc}$, the inclination of the jet axis as derived by the VLBI observations would be a few degrees lower.

After seven years of quiescence, GRO J1655-40 entered a new outburst on February 2005, when the source showed an increase in the X-ray flux [15], optical and near-IR magnitude [23] [1], and a renewed radio activity [18]. The outburst lasted about eight months and has been extensively followed, when possible on a daily basis, at all wavelengths. In March, a state transition occured as GRO J1655-40 entered a thermal state and the radio counterpart faded [7] [19]. In May, the source entered a highly variable, high X-ray luminosity state [8] coupled with a renewed radio emission [20]. GRO J1655-40 then entered a soft state, with no radio detection, and returned to a hard state on September 23 [9]. The source returned to radio activity on September 21 [2].

In this work, we present some preliminary results on a multi-wavelength (radio/infrared/optical/Xray) study of GRO J1655-40 during its 2005 outburst. We focus on the broadband spectral energy distribution during the different stages of the outburst. In particular, using this unprecedented cov- 
erage, and especially thanks to the new constrains given in the mid-IR by Spitzer, we can test the physical self-consistent disk-jet model [14] during the hard state, where the source shows an active radio emission from a compact jet; the Spitzer observations fill the uncovered portion of the previously studied energy broadband spectra of BHs, giving constrains for estimating key parameters of the jet, and ultimately to challenge the jet model assumptions.

\section{Observations}

We have observed the black hole X-ray binary GRO J1655-40 with Spitzer/MIPS during its outburst that started in 2005, following the different stages from the rise of the outburst until quiescence in 2006: 1) in hard state on March 10, 2005, 2) after the first X-ray flux peak on April 6, 2005, 3) after the second and brightest X-ray flux peak, on August 28, 2005, 4) during the decay of the outburst immediately after the BH returns in the hard state, on September 24, 2005 and, finally, 5) after the outburst ended, during quiescence on April 1, 2006. In Fig. 1, we show the X-ray light curve of GRO J1655-40 during the outburst (lower panel) and the $24 \mu \mathrm{m}$ flux densities of the MIPS observations (upper panel). The four Spitzer/MIPS observations during the outburst were all simultaneous with pointed X-ray (RXTE), optical/near-IR (SMARTS) and radio (either with the Very Large Array, VLA, or with the Australia Telescope Compact Array) observations, allowing us to study the evolution of the complete broadband spectrum of the BH during the different stages. GRO J1655-40 was also observed with Spitzer/IRAC in the hard state on September 29, simultaneously with pointed observations of RXTE, SMARTS and quasi-simultaneously (on October 2, 2005) with the VLA. The Spitzer/MIPS observation in quiescence, on April 1, 2006, had no coverage in other wavelength, except for the $2-12 \mathrm{keV}$ observation of the all sky monitor (ASM) onboard RXTE. See Migliari et al. (2007, in prep.), for more detailed information on the observations.

\section{Results and Discussion}

\subsection{The Outburst Evolution}

We can follow the evolution of our six observations during the outburst, using the X-ray light curve (Fig. 1), the HID and the power density spectra (not shown; see Migliari et al. 2007, in prep.), and the spectral energy distributions (Fig. 2).

1) On March 10, 2005 the source was in the hard state. The X-ray flux started already its abrupt rising towards the first peak of the outburst. The power density spectrum show a high rms of $\sim 34 \%$ (the quoted $r m s$ are integrated over $2-15 \mathrm{keV}$ ) and broad features as well as a narrow QPO around $2 \mathrm{~Hz}$, which is typical of a hard state. The X-ray energy spectra show a power law flux of $\sim 77 \%$ the total $2-20 \mathrm{keV}$ flux, which is very close to the limit of $\sim 80 \%$ in the definition of hard state in [17].

2) On April 6, 2005 the source is in a thermal (high/soft) state. The X-ray light curve shows that during this observations GRO J1655-40 was in a steady high flux state, in between the two outburst peaks. The power density spectrum show a $r m s \sim 5 \%$, typical of a thermal state. The disk component in the X-ray spectrum is about $90 \%$ of the total $2-20 \mathrm{keV}$ flux and the source is, accordingly, in the upper-left, soft region of the HID pattern. The radio emission is already 


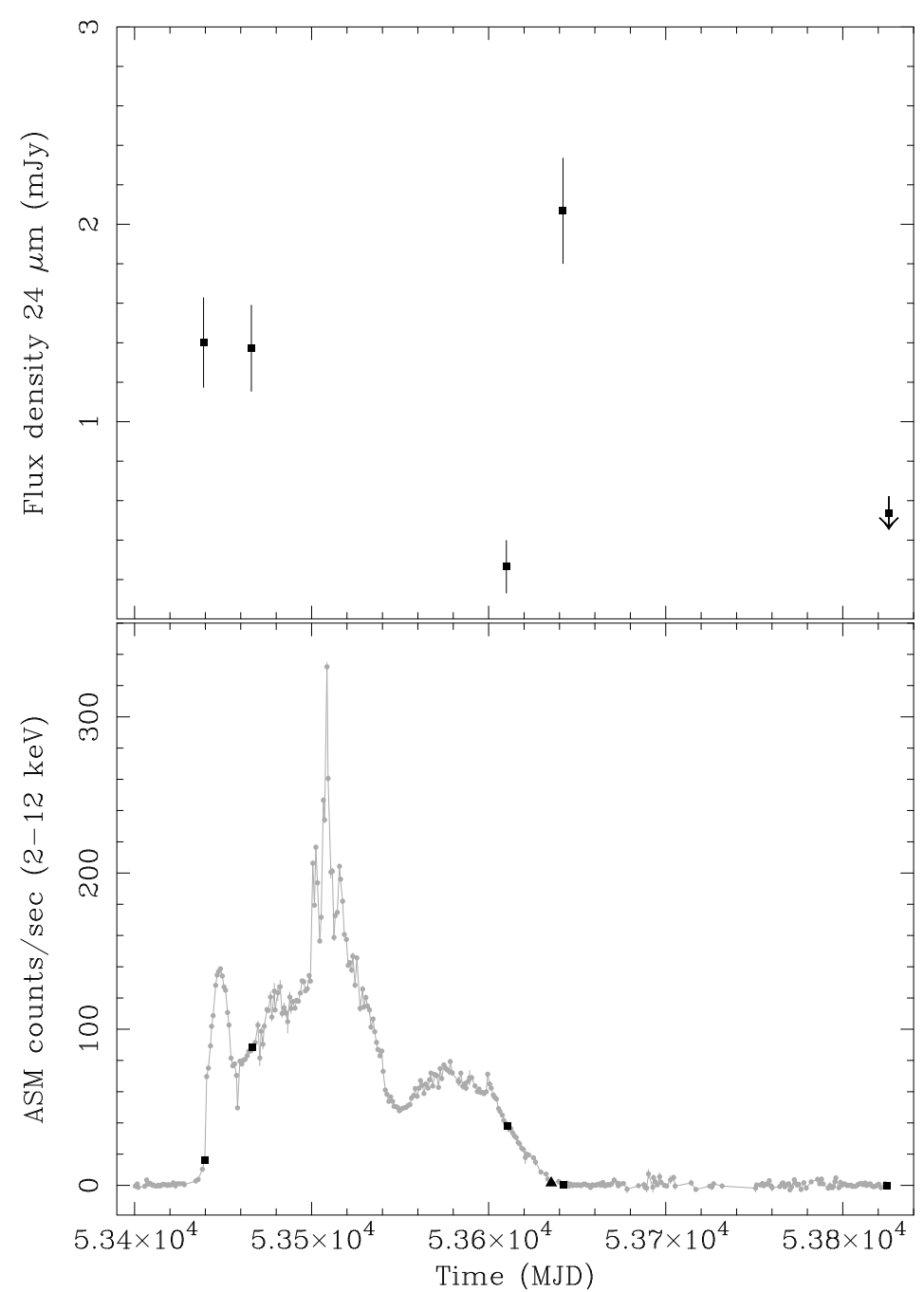

Figure 1: Top panel: Spitzer/MIPS flux densities of GRO J1655-40 during the outburst. Lower panel: RXTE/ASM light curve of the outburst. The squares represent the MIPS observations, the triangle shows the IRAC observation.

quenched and the thermal emission dominates the energy spectrum in the X-ray, optical and midIR bands: Spitzer/MIPS detected the IR tail of the bright disk at $24 \mu \mathrm{m}$. Note also that the hard X-ray component above $\sim 30 \mathrm{keV}$ disappears below the detectable threshold of HEXTE.

3) On August 28, 2005, GRO J1655-40 is in a thermal (high/soft) state. The light curve shows that the source is still at a high flux level, but is starting its decay towards the hard state. The rms noise in the power density spectrum is $\sim 2 \%$, typical of a thermal state. The energy spectrum shows a still-bright disk in the soft X-ray, where the disk flux is still $95 \%$ of the total $2-20 \mathrm{keV}$ flux, and in the optical band, but it shows also the reappeareance of the hard X-ray component above $30 \mathrm{keV}$. The radio emission is not detected yet down to a $3 \sigma$ upper limit of $\sim 1 \mathrm{mJy}$ and the source is only marginally detected at $24 \mu \mathrm{m}$, possibly - if real - the IR tail of the disk component.

4-5) On September 24 and 29, 2005, GRO J1655-40 was observed during the decay of the outburst, when it returned to the hard state. The rms in the power density spectra increased signifi- 


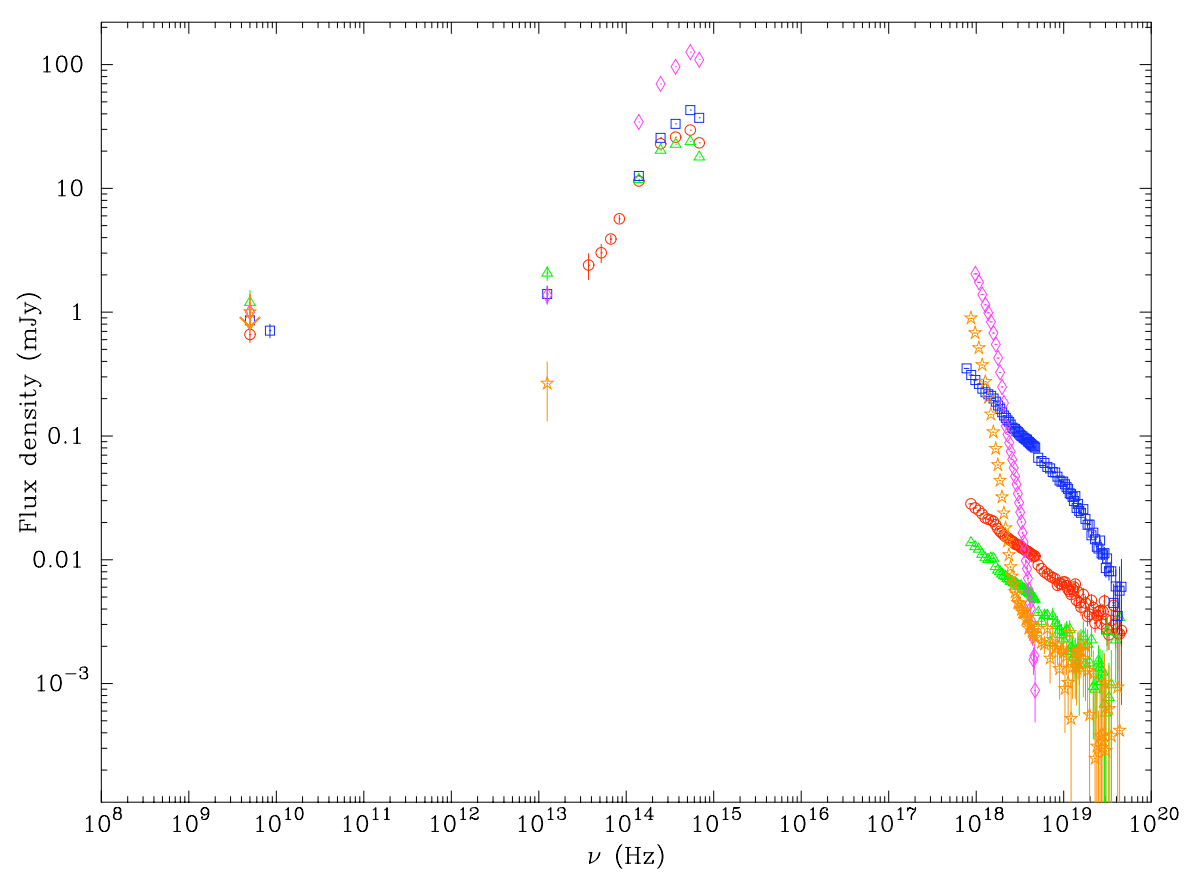

Figure 2: Broadband energy spectra of GRO J1655-40. The different colors and markers show the five spectra under study, with the Spitzer coverage during the outburst: blue squares are the March10 observation (hard state), purple diamonds are April 06, yellow stars are August 28 (thermal state), red circles are September 24 (hard state) and green triangles are September 29 (hard state).

cantly to $\sim 25 \%$ and some features, like a QPO around $0.3 \mathrm{~Hz}$ appears on September 24 . The source reached the bottom right part of the HID and the X-ray spectra are dominated by a non-thermal power-law component whose $2-20 \mathrm{keV}$ flux is about the $91 \%$ of the total flux. The companion star starts probably to contribute to the optical emission. The IR emission (IRAC on September 24 and MIPS on September 29) shows an excess due to the re-brightening of the jet. This jet re-brightening is well visible in the radio band, where its flux density at $5 \mathrm{GHz}$ increases between September 24 and 29 (contrary to the X-ray flux that is still decaying in time).

6) On April 1, 2006 the source has already returned to quiescence; no RXTE/PCA, radio and optical observations are available. The Spitzer/MIPS observations does not detect the source at $24 \mu \mathrm{m}$.

\subsection{Testing the jet model with the SEDs of GRO J1655-40}

\subsubsection{The jet model}

For a detailed discussion of the jet radiative model, we refer the reader to e.g. [13] and [14]. We recall here some fundamental assumptions and a brief description of the model, as outlined in [14]: (1) the total power in the jet scales proportionally with the accretion power at the inner edge of the disk, (2) the jet is expanding freely and, at the very base, only slightly accelerated as a result of 
the pressure gradient, (3) the jet contains cold protons that carries most of the kinetic energy while the leptons most of the radiating energy, (4) particles are eventually accelerated into a power-law distribution, (5) the power law is mantained along the jet beyond the shock region. Geometrically, the base of the jet is attached to the disk at a $r_{0}$ nozzle radius, whose lower limit is the innermost stable orbit of the disk around the black hole. The jet starts as a cilindrical flow, with constant radius $r_{0}$. After this small nozzle region, above $\sim 30$ gravitational radii $\left(r_{g}\right)$, the jet expands sideways at the sound speed for a proton/electon plasma (i.e. $\sim 0.4 c$ ) and is only slightly accelerated by the resulting pressure gradient. At a distance of 100-1000 $r_{g}$ the particles in the jet, that started with a quasi-thermal distribution, are accelerated by a shock into a power-law distribution.

\subsubsection{The fits}

We fitted the energy spectra of September 24 and 28, 2005, using the Interactive Spectral Interpretation System (ISIS [10]). We started the fit by hand outside ISIS, trying to reach a reduced $\chi^{2}$ of less than $\chi_{\text {red }}^{2} \sim 2$. Then we use these parameters as a starting set of parameters for the fit with ISIS. This procedure helps to avoid the automatic minimisation in ISIS falling in false local minima.

The fitting model of our observations consists of three components, corrected for photoelectric absorption: 1) the jet model discussed above, self-consistently integrated with a multi-temperatre disk blackbody, 2) a blackbody with a fixed temperature of $6900 \mathrm{~K}$, to model the companion star, likely a F3-F5 IV, and 3) a disk reflection component with an iron emission line in the range 6.4$7 \mathrm{keV}$. Given the large number of parameters in the model, we have fixed those for which we already have an independent estimate, and those known from previous works to fall in the same range for other BHs (Cyg X-1, GX 339-4; this choice is somewhat arbitrary, but it is a starting point to explore the parameters' space, see [14] for a discussion). We fixed the mass of the $\mathrm{BH}$ to $6.3 \mathrm{M}_{\odot}$ [6] and the distance to its inferred upper limit of $1.7 \mathrm{kpc}$ [5]. We fixed the inclination of the jet to $75^{\circ}$, which is consistent with the jet axis inclination inferred from the radio lobes observations [11], revised with the new lower limit on the distance to the source of $1.7 \mathrm{kpc}$. This value is also consistent with the disk inclination of $\sim 70^{\circ}$ inferred by [6] and allowing a disk-jet misalignment of less than $15^{\circ}$ (e.g. [12]).

\subsubsection{A Comparison with other BHs}

The jet model fit well the data of September 24 , with a $\chi_{\text {red }}^{2}=1.44(78$ d.o.f. $)$, and of September 29, with a $\chi_{\text {red }}^{2}=1.36\left(54\right.$ d.o.f.) (see Fig. 3). In the September 29 fit, although the $\chi^{2}$ statistics seem reasonably good, the model somewhat underestimates the radio emission. The slope of the optically-thick part of the synchrotron spectrum in the modeled jet is steeper than the slope required by the observations. Moreover, the fit finds a solution for a magnetically dominated jet; the equipartition parameter, as defined in [14], is $k \sim 5$, which is different from the value of $k \sim 1$ found for Cyg X-1 and GX 339-4 [14] and for GRO J1655-40 itself on September 24. (Note, however, that there is no reason other than the consistency with previous fits of BHs, to prefer $k \sim 1$ over $k \sim 5$.)

In order to obtain a flatter radio-IR synchrotron spectrum, we let the jet inclination free; the best-fit inclination obtained is $\sim 40^{\circ}$. We would like to stress that, at this stage, the free inclination is meant to be an artificial modification to try to obtain a better fit. A jet inclination of $\sim 40^{\circ}$ seems 

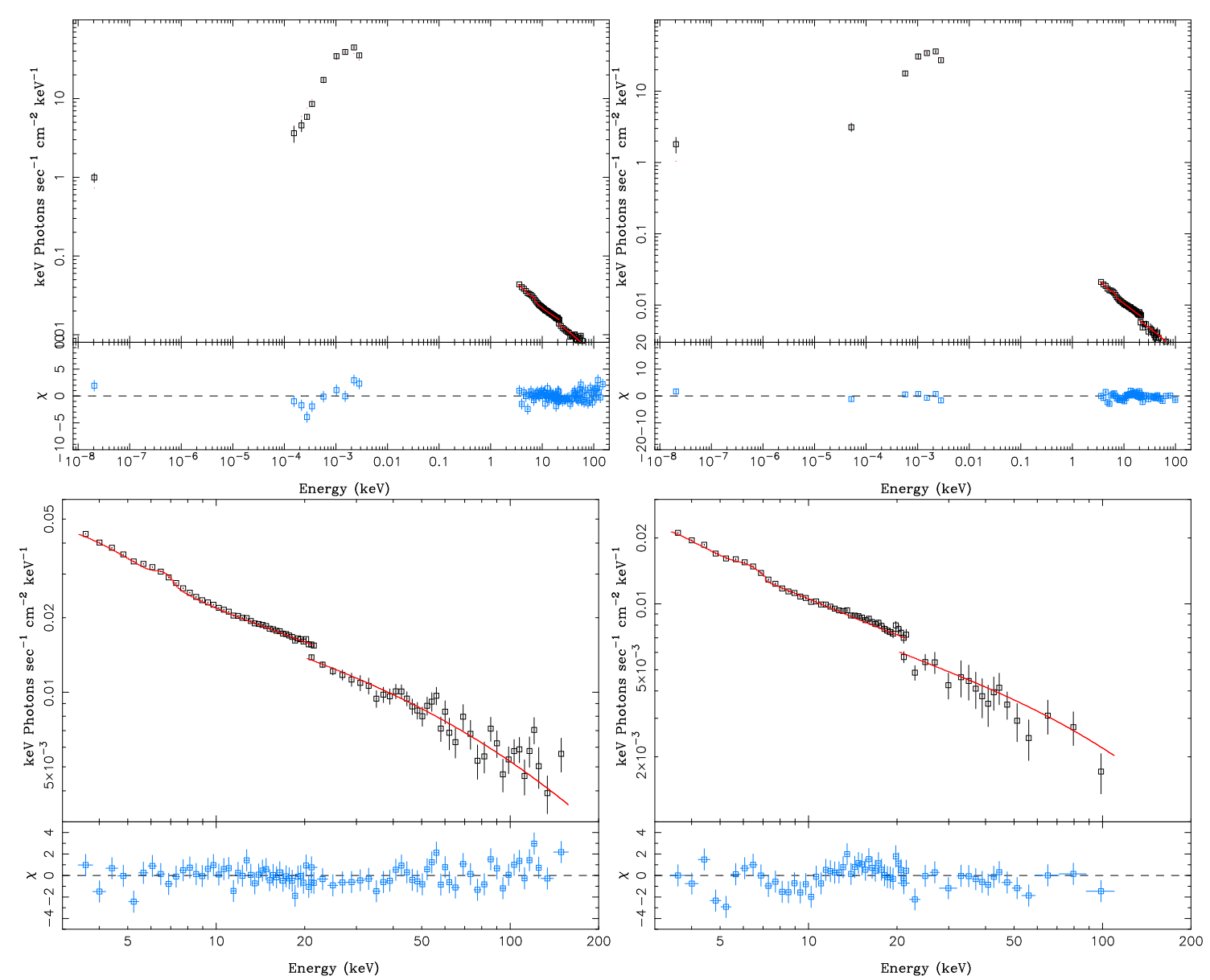

Figure 3: Jet model fits with residuals of the radio-to-X-ray (upper panels) and X-ray spectrum (lower panels) of the September 24 (left) and September 29 (right) observations of GRO J1655-40, with the jet inclination angle fixed to $75^{\circ}$.

unlikely, given a disk inclination of $70^{\circ}[6]$ and the previous estimates of the jet inclination (e.g. [11]). Furthermore, the uncertainties in modeling the jet emission are still too large to attempt an estimate of the jet inclination using these fits. The fit improves slightly as far as the $\chi^{2}$ statistics is concerned, because the statistics is dominated by the X-ray (mainly) and optical part of the spectrum, but we see a by-eye improvement in the fit in the radio band part. As a note, the equipartition parameter is now also consistent with the values found for the September 24 observation and for Cyg X-1 and GX 339-4, where the jet was close to equipartition.

A remarkable result of these fits is that the same model that can fit well other BHs' broadband spectra, seems not to reproduce seemingly well the optically thick part of the synchrotron emission in GRO J1655-40, which is flatter than the model predicts. Possible ways to make the radio-IR emission flatter would be 1) to have a more beamed jet (which is the case emulated in the fit by the smaller jet inclination angle), and 2) to have a more collimated jet, maybe if the side-ways expantion in the post-accelerated jet region, would be somewhat lower than the sound speed in vacuum as assumed in the model. 
As mentioned above, Markoff et al. [14] fitted, using the jet model, some typical hard state observations of the BH XRBs Cyg X-1 and GX 339-4, and discussed the differences and similarities found in the best-fit parameters. Comparing the parameters of GRO J1655-40 with those of the other two BHs, we find that the power that GRO J1655-40 put in to the jet is higher by a factor of $>3$. Also, the nozzle radius is smaller than that of GX 339-4 and very similar to that of Cyg X-1, relfecting the higher X-ray/radio flux ratio. The electron temperature is approximately the same as that found for the other two BHs, as it is the spectral index of the electron distribution. The model finds solutions for a jet close to equipartition in the case of September 24 and of September 29, but in this latter case, only if we let the jet inclination free to adjust the otherwise underestimated radio emission.

The coverage given in the mid-IR by the Spitzer observations, allow us to give more stringent constrains to the broadband emission of BHs, with the ultimate goal of testing/challenging the existing jet models. The analysis of the data is still ongoing, and the results of the complete study will be reported in an upcoming paper.

\section{References}

[1] Buxton, M., Bailyn, C.D., Maitra D., Optical and NIR observations of GRO J1655-40, 2005, ATel, 418

[2] Brocksopp, K., et al., Renewed Radio Emission from GRO J1655-40, 2005, ATel., 612

[3] Fender, R.P., Compact Stellar X-Ray Sources, eds. W.H.G. Lewin and M. van der Klis, 2006, Cambridge University Press, 381

[4] Fender, R.P., Powerful jets from black hole X-ray binaries in low/hard X-ray states, 2001, MNRAS, 322,31

[5] Foellmi C., Depagne E., Dall T. H., Mirabel I. F., On the distance of GRO J1655-40, 2006, A\&A, 457, 249

[6] Greene, J., Bailyn, C.D., Orosz, J.A., Optical and Infrared Photometry of the Microquasar GRO J1655-40 in Quiescence, 2001, ApJ, 554, 1297

[7] Homan, J., GRO J1655-40 rapidly evolving, 2005, ATel, 440

[8] Homan, J., Miller, J.M., Wijnands, R., Lewin, W.H.G., GRO J1655-40 enters a highly-variable, high-luminosity state, 2005, ATel, 487

[9] Homan, J., Miller, J.M., Wijnands, R., Lewin, W.H.G., GRO J1655-40 returning to the hard state, 2005, ATel, 607

[10] Houk J.C., Denicola L.A., ISIS: An Interactive Spectral Interpretation System for High Resolution X-Ray Spectroscopy, 2000, ASPC, 216, 591

[11] Hjellming, R.M., Rupen, M.P., Episodic Ejection of Relativistic Jets by the X-Ray Transient GRO:J1655-40, 1995, Nature, 375, 464

[12] Maccarone T.J., On the misalignment of jets in microquasars, 2002, MNRAS, 336, 1371

[13] Markoff, S., Nowak, M.A., Constraining X-Ray Binary Jet Models via Reflection, 2004, ApJ, 609, 972

[14] Markoff, S., Nowak, M.A., Wilms, J., Going with the Flow: Can the Base of Jets Subsume the Role of Compact Accretion Disk Coronae?, 2005, ApJ, 635, 1216 
[15] Markwardt, C.B., Swank, J.H., New Outburst of GRO J1655-40?, 2005, ATel, 414

[16] McClintock, J.E., \& Remillard, R.A., 2006, Compact Stellar X-Ray Sources, eds. W.H.G. Lewin and M. van der Klis, Cambridge University Press, 157

[17] Remillard R.A., McClintock J.E., X-Ray Properties of Black-Hole Binaries, 2006, ARA\&A, 44, 49

[18] Rupen, M.P., Dhawan, V., Mioduszewski, A.J., Radio detection of GRO J1655-40, 2005, ATel, 419

[19] Rupen, M.P., Dhawan, V., Mioduszewski, A.J., GRO J1655-40 radio counterpart fades, 2005, ATel, 441

[20] Rupen, M.P., Dhawan, V., Mioduszewski, A.J., Recent radio evolution of GRO J1655-40, 2005, ATel, 489

[21] Shaposhnikov N.; Swank, J.; Shrader, C. R.; Rupen, M.; Beckmann, V.; Markwardt, C. B.; Smith, D. A., GRO J1655-40: Early Stages of the 2005 Outburst, 2006, ApJ, in press: astro-ph/0609757

[22] Tingay, S.J., et al., Relativistic Motion in a Nearby Bright X-Ray Source, 1995, Nature, 374, 141

[23] Torres M.A.P., Steeghs, D., Jonker, P., Martini, P., A NIR Observation of GRO J1655-40, 2005, ATel, 417 